\title{
Immunohistochemical expression of cytokeratins 7 and 20 in malignant salivary gland tumors
}

\author{
Nikolaos G Nikitakis ${ }^{2}$ Konstantinos I Tosios², Vasileios S Papanikolaou ${ }^{3}$, Helen Rivera ${ }^{4}$, \\ Stavros I Papanicolaou ${ }^{5}$ and Olga B Ioffe ${ }^{6}$ \\ ${ }^{1}$ Department of Diagnostic Sciences and Pathology, University of Maryland, Baltimore, USA; ${ }^{2}$ Department of \\ Basic Sciences and Oral Biology, Faculty of Dentistry, University of Athens, Greece; ${ }^{3}$ Department of ENT, \\ Leeds General Infirmary, Leeds, UK; ${ }^{4}$ Oral Pathology Laboratory, Faculty of Dentistry, Central University of \\ Venezuela, Venezuela; ${ }^{5}$ Department of Oral Pathology, Faculty of Dentistry, University of Athens, Greece and \\ ${ }^{6}$ Department of Pathology, University of Maryland, Baltimore, USA
}

On the basis of the heterogeneity of cytokeratins 7 and 20 expression in malignant epithelial tumors, the cytokeratin $7 / 20$ immunophenotype has served as a useful diagnostic tool for discrimination of primary and/or metastatic carcinomas of unknown origin. However, the expression pattern of these cytokeratins in malignant salivary gland tumors has not been thoroughly studied. Our study material was composed of 84 malignant tumors of primary major or minor salivary gland origin. Nine histologic types of carcinoma were represented, including mucoepidermoid (26 cases), adenoid cystic (25), polymorphous low grade (11), salivary duct (8), acinic cell (4), ex mixed tumor (3), not otherwise specified (3), clear cell (2), and basal cell (2). In all, 13 cases of primary skin or mucosal squamous cell carcinoma with secondary salivary gland involvement were also examined. Immunoreactivity for cytokeratin 7 was evident in all malignant salivary gland tumors; the staining pattern was diffuse and strong in 62 cases, and focal and strong in 22 cases. In contrast, 78 cases were negative for cytokeratin 20, whereas only six cases (two mucoepidermoid, one adenoid cystic, and three salivary duct) displayed focal weak positivity. Overall, $92.9 \%$ of malignant salivary gland tumors were characterized by a cytokeratin 7 positive / 20 negative immunoprofile, the remaining $7.1 \%$ of cases being positive for both cytokeratins. The latter phenotype was more common in salivary duct carcinomas $(P \leq 0.05)$. On the other hand, most squamous cell carcinomas (69\%) were negative for both cytokeratins, while the remaining cases (31\%) were negative for cytokeratin 20 and focally weakly positive for cytokeratin 7 . We suggest that assessment of cytokeratin $7 / 20$ immunoprofile may facilitate the differential diagnosis of (a) primary malignant salivary gland tumors from metastatic tumors, (b) metastatic salivary gland tumors, (c) primary salivary gland tumors, especially mucoepidermoid carcinomas, from squamous cell carcinomas, and (d) salivary duct carcinomas from other malignant salivary gland tumors.

Modern Pathology (2004) 17, 407-415, advance online publication, 13 February 2004; doi:10.1038/modpathol.3800064

Keywords: salivary gland tumors; Squamous cell carcinoma; Cytokeratins 7 and 20; Immunohistochemistry

The cytokeratins (CKs) constitute a family of intermediate filament proteins whose expression is generally confined to epithelia and their neoplasms. ${ }^{1,2}$ At least 20 different CKs have been described, which exhibit a highly diverse expression in various epithelia. ${ }^{1,2}$ Based on the observation

Correspondence: NG Nikitakis, DDS, PhD, Department of Diagnostic Sciences and Pathology, Room 4-C-02, Dental School, University of Maryland, Baltimore, 666 West Baltimore Street, Baltimore, MD 21201, USA.

E-mail: nin001@ dental.umaryland.edu

Received 14 August 2003; revised 15 December 2003; accepted 16 December 2003; published online 13 February 2004 that carcinomas largely reserve the CK profile of their epithelium of origin, differential immunohistochemical staining for specific CKs may aid in the accurate identification and classification of different types of carcinomas. ${ }^{2}$ In this context, the diverse expression pattern of CK7 and CK20 among epithelial tumors has been reported as a useful diagnostic marker for discriminating primary from metastatic carcinomas of various origins. ${ }^{3}$

The expression profile of CK7 and CK20 has been thoroughly studied in a range of carcinomas. ${ }^{3-16}$ CK7 expression is seen in most carcinomas, with the exception of tumors arising from the colon, prostate, kidney, and thymus; carcinoid tumors of the lung 
and gastrointestinal tract origin; and Merkel cell tumors of the skin. ${ }^{4-6}$ On the other hand, CK20 positivity has been detected in the vast majority of adenocarcinomas of the colon, mucinous ovarian tumors, and Merkel cell carcinomas and frequently in transitional cell carcinomas and adenocarcinomas of the stomach, bile duct system, and pancreas. ${ }^{5-8}$ Interestingly, most squamous cell carcinomas, in general, are negative for both CK7 and CK20, with the exception of cervical squamous cell carcinomas, which are frequently positive for CK7. ${ }^{6}$ While assessment of a single CK is of limited diagnostic usefulness, knowledge of the combined CK7/CK20 immunohistochemical profile of specific tumor types has been proven valuable for identifying the tissue of origin of carcinomas of unknown origin, a finding that bears prognostic and therapeutic significance. ${ }^{3}$ Characteristic examples include the differential diagnosis between lung, endometrial, nonmucinous ovarian, and breast adenocarcinomas (predominantly CK7 + /CK20-) and colon adenocarcinoma (mainly CK7-/CK20 + ), ${ }^{6,9-12}$ or the distinction of the CK20 + Merkel cell carcinoma of skin and CK20 + small cell carcinoma of salivary glands from the CK20- small cell carcinomas of other origins. ${ }^{6-8,13}$

Carcinomas of salivary gland origin represent an important subset of malignant epithelial tumors. These tumors can metastasize to distant sites and should be included in the differential diagnosis of metastatic tumors of unknown primary. ${ }^{17}$ Moreover, diagnostic difficulties arise when carcinomas from remote sites metastasize to salivary glands ${ }^{18}$ or when squamous cell carcinomas of mucosal or skin origin metastasize to or invade the salivary glands. ${ }^{19}$ Finally, the overlapping histopathologic features of the numerous types of malignant salivary gland tumors often pose difficulties in the accurate classification of these neoplasms, which may exhibit a wide range of biologic behaviors. ${ }^{20}$ In all these instances, assessment of the CK profile could facilitate the precise diagnosis of malignant salivary gland tumors. It was the purpose of our study to assess the CK7/CK20 expression patterns of malignant salivary gland tumors, which has not been hitherto thoroughly investigated.

\section{Materials and methods}

In total, 84 consecutive cases of primary malignant salivary gland tumors were retrieved from the surgical pathology files of the Department of Pathology, University of Maryland Medical System, Baltimore, USA (33 cases), the Department of Oral Pathology and Surgery, University of Athens, Greece (39 cases), and the Oral Pathology Laboratory, Faculty of Dentistry, Central University of Venezuela, Caracas, Venezuela (12 cases). A total of 52 cases originated from minor salivary glands, 30 from major salivary glands, while the site was unspeci- fied in two cases. Of 83 patients with available gender information, 35 were male and 48 were female. Age information was available for 83 patients, the mean age of which was 53.5 years (range 5-88 years). The most common histologic type was mucoepidermoid carcinoma (26 cases including nine low-grade, 12 intermediate-grade, and five high-grade tumors), followed by adenoid cystic carcinoma (25 cases), polymorphous lowgrade adenocarcinoma (11 cases), salivary duct carcinoma (eight cases), acinic cell carcinoma (four cases), carcinoma ex mixed tumor (three cases), adenocarcinoma not otherwise specified (three cases), basal cell adenocarcinoma (two cases), and clear cell adenocarcinoma (two cases); both cases of clear cell adenocarcinoma represented primary salivary gland tumors in the absence of a clear cell tumor elsewhere in the body.

In all, 13 cases of squamous cell carcinoma secondarily involving the salivary glands (by means of direct extension or metastasis from primary skin or mucosal tumors of the head and neck) were also included in the study. Nine patients were female and four were male. The mean age of the 11 patients with available information was 63.8 years (range 4679 years).

Hematoxylin-and-eosin-stained slides were reviewed by two pathologists (NGN and OBI) and the original diagnoses were confirmed. One representative section of each case was immunostained for CK7 (NeoMarkers, Union City, CA, USA) and CK20 (DAKO, Glostrup, Denmark). In essence, 5- $\mu \mathrm{m}$ thick sections of paraffin-embedded tissue were deparaffinized and rehydrated. An antigen retrieval procedure was performed by placing the sections in $200 \mathrm{ml}$ of Target Retrieval Solution, pH 6.0 (DAKO, Carpinteria, CA, USA), for $20 \mathrm{~min}$ at $100^{\circ} \mathrm{C}$. After cooling for $20 \mathrm{~min}$, endogenous peroxidase activity was blocked with 3\% hydrogen peroxide. Sections were subsequently labeled with the aforementioned primary antibodies using an automated slide stainer (Ventana ES Slide Staining System, Tucson, AZ, USA). The sections were incubated with a secondary biotinylated antibody (VIEW DAB Detection Kit, Ventana). A streptavidin horseradish peroxidase (Ventana) was applied and the specimens were stained with diaminobenzidine (Ventana) and counterstained with Harris hematoxylin. To assure proper staining, appropriate controls were included in each run. Positive controls included known positive cases for each antibody (CK7 + gestational endometrium and CK20 + colon adenocarcinoma, respectively), while negative controls were processed otherwise identically, but the primary antibody was omitted.

To evaluate and compare the results of the immunohistochemistry, the immunostaining was scored semiquantitatively in the entire tumor. Only cases exhibiting greater than $5 \%$ positive tumor cells were considered as positive. ${ }^{6}$ Both positivity (focal or diffuse, defined as less than $25 \%$ or more 
than $25 \%$ positive tumor cells, respectively) and intensity (weak or strong) of staining were recorded. Moreover, the distribution of staining in the different cellular compartments of the various tumors was recorded.

The frequency of different CK7/CK20 immunohistochemical profiles among the various subtypes of the studied tumors was compared by means of $\chi^{2}$ statistical test (Minitab ${ }^{\mathbb{R}}$ statistical package).

\section{Results (Table 1)}

\section{Malignant Salivary Gland Tumors}

All malignant salivary gland tumors (84 of 84, $100 \%$ ) were positive for CK7. The intensity of the immunostaining was strong in all tumors (Figures 1-4). Concerning the positivity of staining, the majority of cases (65 of 84, 77.4\%) exhibited diffuse positivity, while 19 of 84 cases $(22.6 \%)$ were focally positive. Mucoepidermoid carcinomas were diffusely CK7 positive in 24 of 26 cases (92.3\%), only two cases $(7.7 \%)$, both classified as intermediate-grade tumors, showing focal staining. Focal positive CK7 staining was also seen in two of three cases $(66.7 \%)$ of carcinomas ex mixed tumors and 11 of 25 cases $(44 \%)$ of adenoid cystic carcinomas, while it accounted for $0-33.3 \%$ of cases among the other types of malignant salivary gland tumors.

On the other hand, only six of 84 cases $(7.1 \%)$ of malignant salivary gland tumors were positive for CK20, including two of 26 cases $(7.7 \%)$ of mucoepidermoid carcinomas (including one low-grade and one intermediate-grade tumor), one of 25 cases $(4 \%)$ of adenoid cystic carcinomas, and three of eight cases $(37.5 \%)$ of salivary duct carcinomas (Figures 1-4). All CK20-positive cases showed focal positivity and weak intensity of the immunostaining, which however was not seen in the corresponding negative controls. Overall, a CK7 +/CK20immunohistochemical profile was seen in $78 / 84$ cases $(92.9 \%)$ of malignant salivary gland tumors. The remaining six tumors $(7.1 \%)$ demonstrated a CK7 + /weak focal CK20 + profile, while the CK7-/ $\mathrm{CK} 20+$ and CK7-/CK20- immunophenotypes were not expressed by any studied malignant salivary gland tumor.

Given the heterogeneity of the cell subtypes (eg acinar, luminal, or myoepithelial cells) that constitute the tumor cell population of the various types of

Table 1 CK7/CK20 immunohistochemical profile in malignant salivary gland tumors and squamous cell carcinomas

\begin{tabular}{|c|c|c|c|c|c|c|}
\hline Histologic type & CK7-positive cases & CK20-positive cases & CK7+/CK20+ & CK7+/CK20- & CK7-/CK20+ & CK7-/CK20- \\
\hline MEC & $\begin{array}{c}26 / 26 \\
(24 \mathrm{DS}, 2 \mathrm{FS})\end{array}$ & $\begin{array}{c}2 / 26 \\
(2 \mathrm{FW})\end{array}$ & $\begin{array}{c}2 / 26 \\
(7.7 \%)\end{array}$ & $\begin{array}{c}24 / 26 \\
(92.3 \%)\end{array}$ & $\begin{array}{l}0 / 26 \\
(0 \%)\end{array}$ & $\begin{array}{l}0 / 26 \\
(0 \%)\end{array}$ \\
\hline AdCC & $\begin{array}{c}25 / 25 \\
\text { (14 DS, } 11 \text { FS) }\end{array}$ & $\begin{array}{c}1 / 25 \\
(1 \mathrm{FW})\end{array}$ & $\begin{array}{l}1 / 25 \\
(4 \%)\end{array}$ & $\begin{array}{l}24 / 25 \\
(96 \%)\end{array}$ & $\begin{array}{l}0 / 25 \\
(0 \%)\end{array}$ & $\begin{array}{l}0 / 25 \\
(0 \%)\end{array}$ \\
\hline PLGA & $\begin{array}{c}11 / 11 \\
(10 \mathrm{DS}, 1 \mathrm{FS})\end{array}$ & $0 / 11$ & $\begin{array}{l}0 / 11 \\
(0 \%)\end{array}$ & $\begin{array}{c}11 / 11 \\
(100 \%)\end{array}$ & $\begin{array}{l}0 / 11 \\
(0 \%)\end{array}$ & $\begin{array}{l}0 / 11 \\
(0 \%)\end{array}$ \\
\hline SDC & $\begin{array}{c}8 / 8 \\
(7 \mathrm{DS}, 1 \mathrm{FS})\end{array}$ & $\begin{array}{c}3 / 8 \\
(3 \mathrm{FW})\end{array}$ & $\begin{array}{c}3 / 8 \\
(37.5 \%)\end{array}$ & $\begin{array}{c}5 / 8 \\
(62.5 \%)\end{array}$ & $\begin{array}{c}0 / 8 \\
(0 \%)\end{array}$ & $\begin{array}{c}0 / 8 \\
(0 \%)\end{array}$ \\
\hline ACC & $\begin{array}{c}4 / 4 \\
(3 \mathrm{DS}, 1 \mathrm{FS})\end{array}$ & $0 / 4$ & $\begin{array}{c}0 / 4 \\
(0 \%)\end{array}$ & $\begin{array}{c}4 / 4 \\
(100 \%)\end{array}$ & $\begin{array}{c}0 / 4 \\
(0 \%)\end{array}$ & $\begin{array}{c}0 / 4 \\
(0 \%)\end{array}$ \\
\hline Ca-ex-MT & $\begin{array}{c}3 / 3 \\
(1 \mathrm{DS}, 2 \mathrm{FS})\end{array}$ & $0 / 3$ & $\begin{array}{c}0 / 3 \\
(0 \%)\end{array}$ & $\begin{array}{c}3 / 3 \\
(100 \%)\end{array}$ & $\begin{array}{c}0 / 3 \\
(0 \%)\end{array}$ & $\begin{array}{c}0 / 3 \\
(0 \%)\end{array}$ \\
\hline NOS & $\begin{array}{c}3 / 3 \\
(2 \mathrm{DS}, 1 \mathrm{FS})\end{array}$ & $0 / 3$ & $\begin{array}{c}0 / 3 \\
(0 \%)\end{array}$ & $\begin{array}{c}3 / 3 \\
(100 \%)\end{array}$ & $\begin{array}{c}0 / 3 \\
(0 \%)\end{array}$ & $\begin{array}{c}0 / 3 \\
(0 \%)\end{array}$ \\
\hline CCC & $\begin{array}{c}2 / 2 \\
(2 \mathrm{DS})\end{array}$ & $0 / 2$ & $\begin{array}{c}0 / 2 \\
(0 \%)\end{array}$ & $\begin{array}{c}2 / 2 \\
(100 \%)\end{array}$ & $\begin{array}{c}0 / 2 \\
(0 \%)\end{array}$ & $\begin{array}{c}0 / 2 \\
(0 \%)\end{array}$ \\
\hline BCAC & $\begin{array}{c}2 / 2 \\
(2 \mathrm{DS})\end{array}$ & $0 / 2$ & $\begin{array}{c}0 / 2 \\
(0 \%)\end{array}$ & $\begin{array}{c}2 / 2 \\
(100 \%)\end{array}$ & $\begin{array}{c}0 / 2 \\
(0 \%)\end{array}$ & $\begin{array}{c}0 / 2 \\
(0 \%)\end{array}$ \\
\hline All MSGT & $\begin{array}{c}84 / 84 \\
\text { (65 DS, } 19 \text { FS) }\end{array}$ & $\begin{array}{c}6 / 84 \\
(6 \mathrm{FW})\end{array}$ & $\begin{array}{c}6 / 84 \\
(7.1 \%)\end{array}$ & $\begin{array}{c}78 / 84 \\
(92.9 \%)\end{array}$ & $\begin{array}{l}0 / 84 \\
(0 \%)\end{array}$ & $\begin{array}{l}0 / 84 \\
(0 \%)\end{array}$ \\
\hline SCCa & $\begin{array}{c}4 / 13 \\
(4 \mathrm{FW})\end{array}$ & $0 / 13$ & $\begin{array}{l}0 / 13 \\
(0 \%)\end{array}$ & $\begin{array}{c}4 / 13 \\
(30.8 \%)\end{array}$ & $\begin{array}{l}0 / 13 \\
(0 \%)\end{array}$ & $\begin{array}{c}9 / 13 \\
(69.2 \%)\end{array}$ \\
\hline
\end{tabular}

Abbreviations: MSGT, malignant salivary gland tumor; SCCa, squamous cell carcinoma; MEC, mucoepidermoid carcinoma; AdCC, adenoid cystic carcinoma; PLGA, polymorphous low-grade adenocarcinoma; SDC, salivary duct carcinoma; ACC, acinic cell carcinoma; Ca-ex-MT, carcinoma ex mixed tumor; NOS, adenocarcinoma not otherwise specified; CCC, clear cell carcinoma; BCAC, basal cell adenocarcinoma; DS, diffuse strong; FS, focal strong; FW, focal weak. 

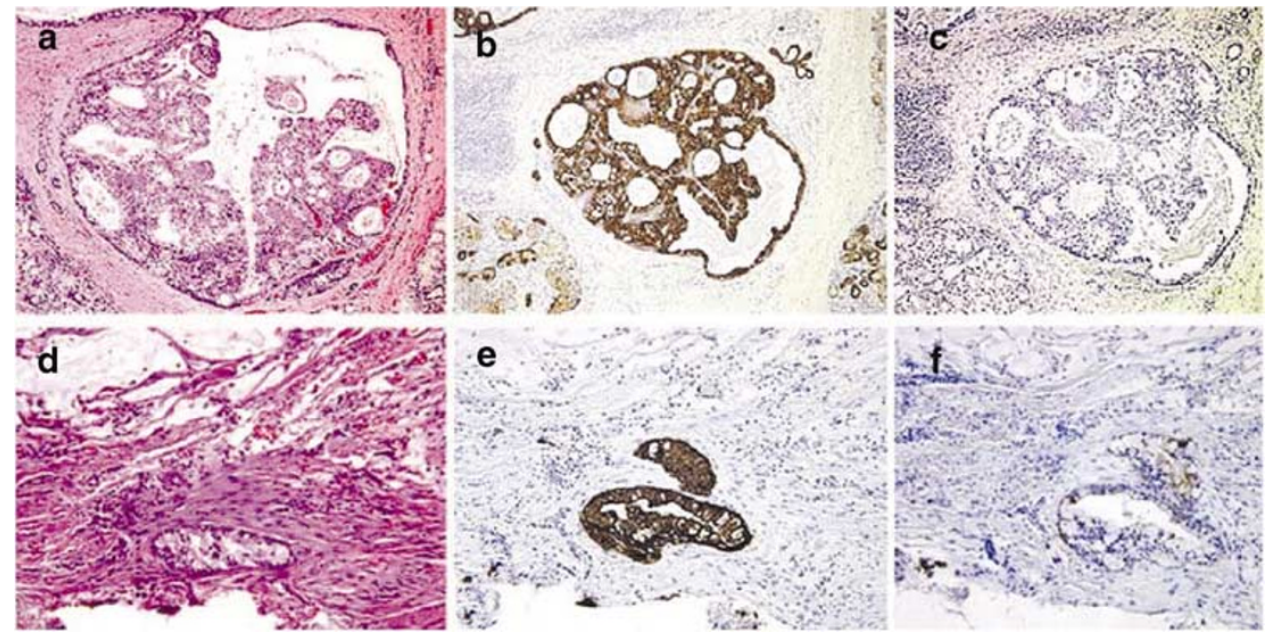

Figure 1 Hematoxylin and eosin appearance (a), (d), and immunohistochemical analysis for CK7 (b), (e) and CK20 (c), (f) in two cases of mucoepidermoid carcinoma. The first case (upper panel) showed diffuse and strong positivity for CK7, while it was negative for CK20. The second case (lower panel) was diffusely and strongly positive for CK7, also demonstrating weak and focal expression of CK20. Magnification for $(\mathbf{a}-\mathbf{c})$ is $\times 100$, while magnification for $(\mathbf{d}-\mathbf{f})$ is $\times 200$.

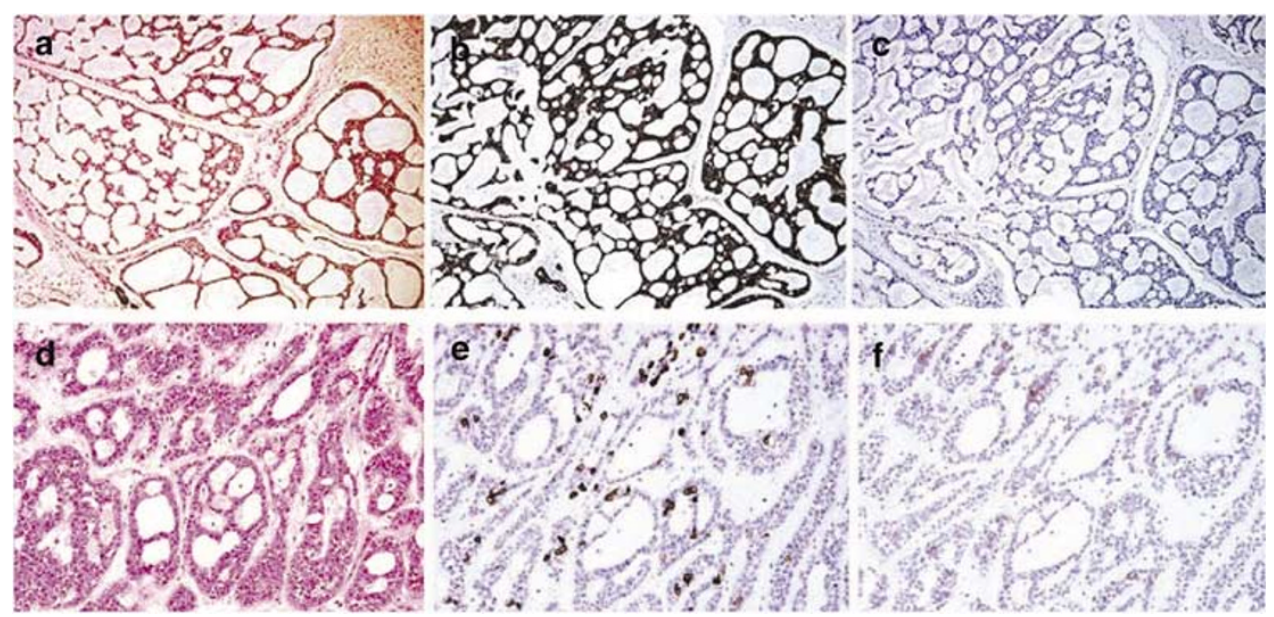

Figure 2 Hematoxylin and eosin appearance (a), (d), and immunohistochemical analysis for CK7 (b), (e) and CK20 (c), (f) in two cases of adenoid cystic carcinoma. The first case (upper panel) displayed diffuse and strong positivity for CK7 (b), being negative for CK20 (c). The second case (lower panel) showed focal and strong CK7 expression (e), and focal and weak CK20 expression (f). Magnification for (ac) is $\times 100$, while magnification for $(\mathbf{d}-\mathbf{f})$ is $\times 200$.

malignant salivary gland tumors, the distribution of the staining in the cellular compartments of tumors was also assessed. Noteworthy is that surrounding normal salivary glands showed diffuse positivity for CK7, which was stronger in luminal ductal cells and less intense in basal ductal, myoepithelial, and acinar cells. In contrast, all cellular elements of normal salivary glands were negative for CK20.

In cases showing diffuse positivity for CK7, all, or almost all, tumor cells were positive, independent of their subtype. In contrast, tumors characterized by focal positivity for CK7 demonstrated a preferential staining of particular subtypes of the tumor cells. More specifically, focally staining mucoepidermoid carcinomas showed positivity of the mucous, intermediate, and luminal cells, but not of the epider- moid cells. In focally positive adenoid cystic carcinomas, the immunoreactivity was limited to the luminal cells, while expression in myoepithelial cells was very weak or negative. Similar to the distribution pattern seen in adenoid cystic carcinomas, focally positive carcinomas ex mixed tumor showed preferential staining of the luminal cells, with weak or negative results in the myoepithelial component. Focally positive cases of polymorphous low-grade adenocarcinoma and acinic cell carcinoma showed positivity limited to luminal and nonacinar cells, respectively. Finally, focally staining salivary duct carcinomas and adenocarcinomas not otherwise specified did not exhibit a preferential staining pattern of a specific cell type, while all studied clear cell carcinomas and basal cell adeno- 


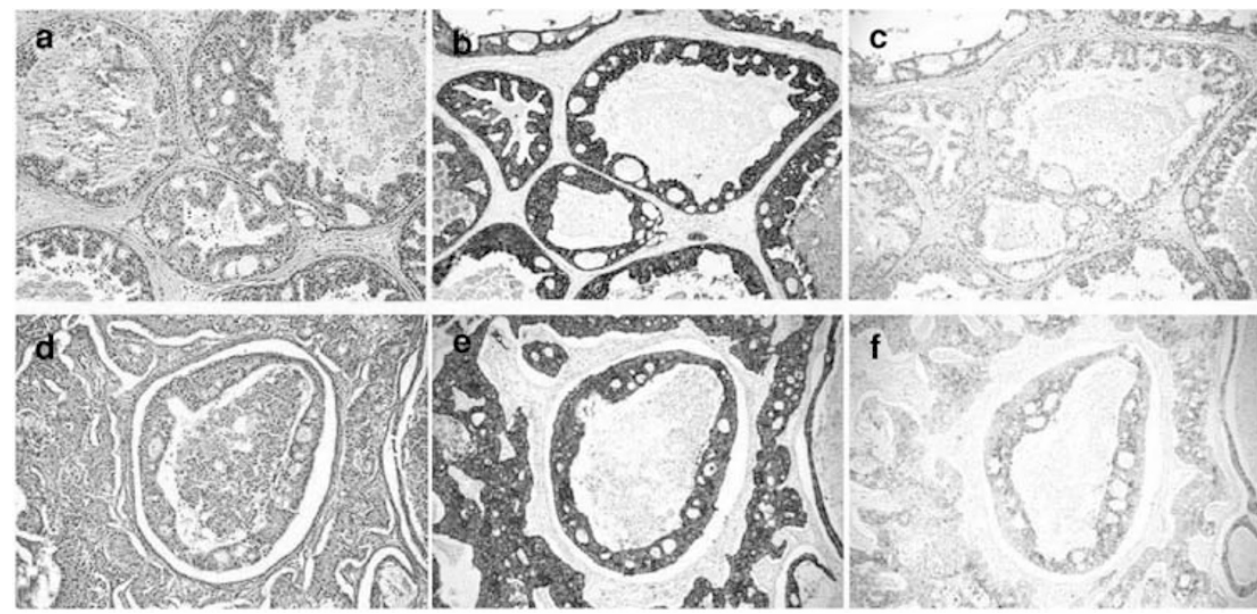

Figure 3 Hematoxylin and eosin appearance (a), (d), and immunohistochemical analysis for CK7 (b), (e) and CK20 (c), (f) in two cases of salivary duct carcinoma. Both cases demonstrated diffuse and strong CK7 expression. However, the first case (upper panel) did not show staining for CK20, which was focally and weakly expressed in the second case (lower panel). Magnification for all photographs is $\times 100$.

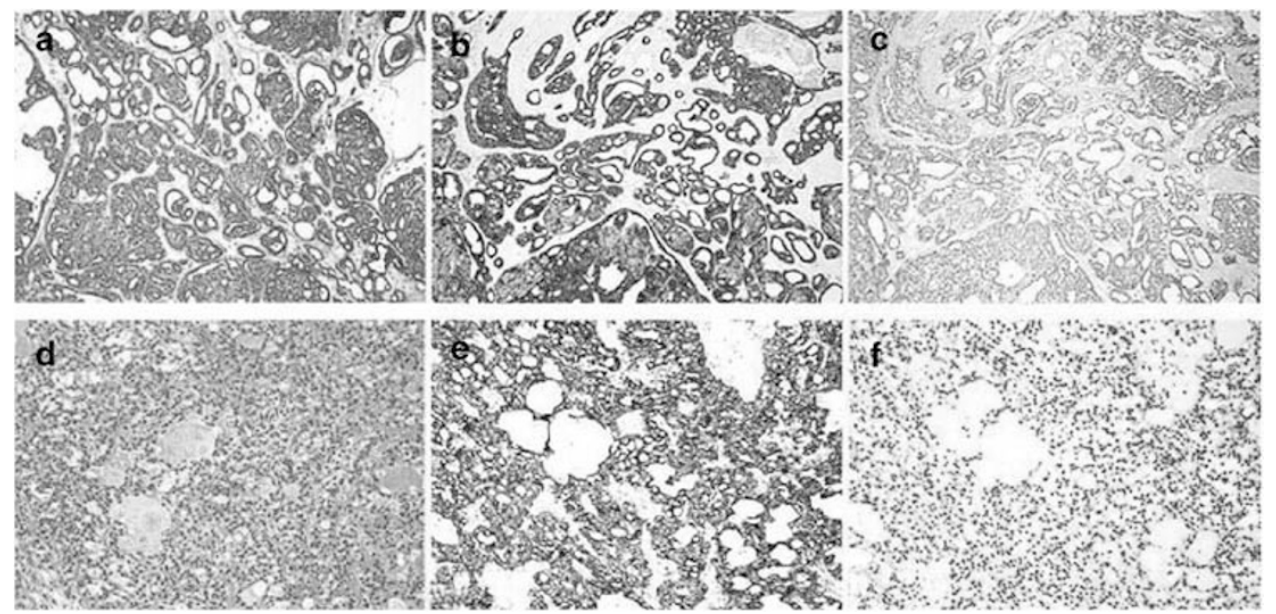

Figure 4 Hematoxylin and eosin appearance (a), (d), and immunohistochemical analysis for CK7 (b), (e) and CK20 (c), (f) in polymorphous low-grade adenocarcinoma (upper panel) and acinic cell carcinoma (lower panel). In both cases, a typical diffuse and strong CK7 positivity is depicted, in the absence of CK20 expression. Magnification for $(\mathbf{a}-\mathbf{c})$ is $\times 100$, while magnification for (d-f) is $\times 200$.

carcinomas were diffusely positive. On the other hand, CK20-positive cases, which uniformly showed focal and weak positivity, did not display any identifiable pattern of distribution in favor of a particular cell subtype.

\section{Squamous Cell Carcinomas}

Four of 13 cases $(30.8 \%)$ of squamous cell carcinoma were positive for CK7 (Figure 5). All positive cases exhibited focal positivity (in three of them limited to less than $10 \%$ of the tumor cell population) and weak intensity of the immunostaining. All squamous cell carcinomas were negative for CK20 (Figure 5). Overall, a CK7-/CK20- immunohistochemical profile was predominant, seen in $9 / 13$ cases $(69.2 \%)$ of squamous cell carcinoma. Focally weakly CK7 +/CK20- immunophenotype ac- counted for the remaining four tumors $(30.8 \%)$, while none of the studied squamous cell carcinomas demonstrated a CK7 + /CK20 + or a CK7-/CK20 + immunophenotype.

\section{Statistical Analysis}

The frequency of CK7 + /CK20- immunophenotype was significantly higher in malignant salivary gland tumors as a group compared to squamous cell carcinomas $(P \leq 0.05)$. When histologic subtypes of malignant salivary gland tumors were examined separately, each subtype showed a higher frequency of this phenotype compared to squamous cell carcinomas $(P \leq 0.05)$, with the exception of salivary duct carcinomas, for which there was no statistically significant difference from squamous cell carcinomas. 


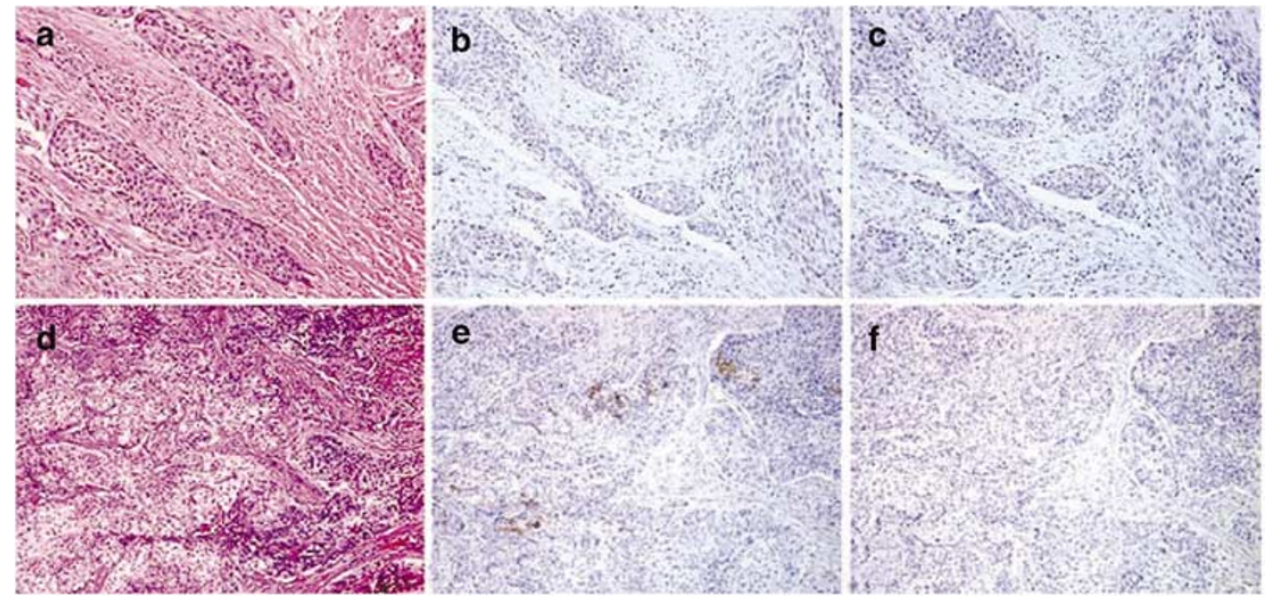

Figure 5 Hematoxylin and eosin appearance (a), (d), and immunohistochemical analysis for CK7 (b), (e) and CK20 (c), (f) in two cases of squamous cell carcinoma. The first case (upper panel) is negative for both markers. The second case (lower panel) is focally and weakly positive for CK7 in the absence of CK20 expression. Magnification for $(\mathbf{a}-\mathbf{c})$ is $\times 200$, while magnification for $(\mathbf{d}-\mathbf{f})$ is $\times 100$.

A CK7 + /CK20 + immunoprofile was recorded more often in salivary duct carcinomas compared to all other malignant salivary gland tumors $(P \leq 0.05)$. This statistical significance persisted even when salivary duct carcinomas were compared to other tumors with an occasional CK7 + /CK20 + phenotype, that is, mucoepidermoid carcinomas and adenoid cystic carcinomas.

\section{Discussion}

In this study, we evaluated the CK7 and CK20 profile of malignant salivary gland tumors in order to identify patterns of expression that could facilitate the distinction of these tumors from primary or metastatic carcinomas of other origin, including squamous cell carcinoma, and the discrimination between malignant salivary gland tumors of different histopathologic type. Although several investigators have examined the expression of CKs in neoplastic lesions of salivary glands, ${ }^{19,21-31}$ to the best of our knowledge this is the first time that the combined CK7 and CK20 profile of malignant salivary gland tumors is analyzed in a sizeable group of these tumors.

Previous investigations have addressed the expression of a diverse array of CKs in normal salivary glands. Such studies have shown that different CKs are localized in the various compartments of normal salivary glands, for example, CK8 and CK18 are expressed in the luminal duct and acinar cells, while CK5 and CK14 are primarily seen in basal duct cells and myoepithelial cells. ${ }^{21-24}$ Interestingly, CK7 has been shown to be expressed to a different degree in the various epithelial salivary gland elements; more specifically, the luminal cells of the salivary ducts are strongly positive for CK7, while the acinar, basal, and myoepithelial cells stain with less intensity or, according to some investigators, not at all. ${ }^{21-24}$ On the other hand, CK20 has been reported to be consistently absent from all salivary gland epithelial elements. ${ }^{22,24,25}$

The pattern of CK expression in normal salivary glands has been demonstrated to be recapitulated to some extent in their benign and malignant neoplasms. Nonetheless, differences among tumors of different histopathologic type, different tumors of the same type, or even different areas of the same tumor were evident. In these studies, CK7 or, more rarely, CK20 were included as part of a broader immunohistochemical panel, usually applied to one or only few of the numerous subgroups of salivary gland tumors. In general, CK7 was reported to be expressed by the vast majority of studied salivary gland tumors, including benign and malignant tumors. ${ }^{19,22-24,26-31}$ These results are in accordance with our findings showing that all cases of malignant salivary gland tumors were positive for CK7, despite differences in intensity, positivity, and distribution of staining. Regarding the latter, the preferential CK7 immunohistochemical expression in certain subtypes of tumor cells observed in our study, such as luminal over myoepithelial cells in adenoid cystic carcinomas, agrees with the findings of other authors and partially reiterates the distribution of CK7 expression in the different cellular compartments of normal salivary glands.

On the other hand, CK20 has been studied in a limited number of benign and malignant salivary gland tumors. ${ }^{7,22,24,25,28,29}$ In these investigations, CK20 was found to be negative in malignant salivary gland tumors, with the exception of sporadic cases of mucoepidermoid carcinomas, ${ }^{24}$ carcinomas ex mixed tumor, ${ }^{28}$ and mucinous adenocarcinoma, ${ }^{29}$ which primarily showed a focal positivity pattern. Again, these results are in agreement with the negative CK20 staining in the vast majority of 
malignant salivary gland tumors in our study and also correspond to the absence of CK20 expression in normal salivary glands.

The current study did not find significant differences in the CK7/CK20 immunohistochemical profile among the different histologic types of salivary gland carcinomas, which could help in their discrimination. Salivary duct carcinoma was the only exception to this rule, showing a higher frequency of $\mathrm{CK} 7+/ \mathrm{CK} 20+$ immunophenotype compared to all and each one of the rest of malignant salivary gland tumors. However, the fact that other malignant salivary gland tumors, specifically mucoepidermoid carcinomas and adenoid cystic carcinomas, could occasionally demonstrate a CK7 + / CK20 + profile weakens the diagnostic significance of this observation. It is also worth noting that although all malignant salivary gland tumors were positive for CK7, the distribution of the staining varied among cases. In this context, the frequent occurrence of CK7 focal staining in adenoid cystic carcinoma compared to the almost universally diffuse pattern in polymorphous low-grade adenocarcinoma may also be of some diagnostic usefulness in discriminating these tumors, which often present with overlapping morphologic features. ${ }^{32}$

Primary tumors of various origin and histologic type may metastasize to the salivary glands, posing significant diagnostic and therapeutic problems. ${ }^{18}$ Immunohistochemical stains for CK7 and CK20 can add valuable information for the differential diagnosis of primary salivary gland carcinomas vs metastatic carcinomas to the head and neck. Based on their consistent CK7 + /CK20- (or, rarely, weekly focally CK20 + ) immunohistochemical phenotype, malignant salivary gland tumors are similar to carcinomas of breast, lung, endometrium, ovaries, and thyroid gland. Therefore, metastatic tumors from these organs to the head and neck region cannot be distinguished from primary malignant salivary gland tumors on the basis of CK7/CK20 immunoprofile. Nonetheless, the relatively common CK20 positivity among salivary duct carcinomas may help distinguish them from morphologically similar carcinomas of breast or lung origin. In contrast, identification of a CK7 + profile in malignant salivary gland tumors may considerably aid in their discrimination from tumors with a predominant CK7-/CK20 + immunophenotype, such as colorectal carcinoma, or a prevalent CK7-/CK20profile, such as adrenal cortical, prostatic, and renal cell carcinomas. A CK7 + /CK20 + immunohistochemical profile, although much more common in pancreatic carcinomas, cholangiocarcinomas, and bladder transitional carcinomas, can also be seen in sporadic cases of malignant salivary gland tumors, especially salivary duct carcinomas, thus undermining its diagnostic usefulness. However, a diffuse and/or strong CK20 + phenotype in a carcinoma would militate against a primary salivary gland origin. Finally, it should be kept in mind that small cell carcinomas of salivary gland origin, although not included in our study, have been reported to display a frequent CK20 diffuse positivity, similar to Merkel cell carcinomas of skin, which discriminates them from small cell carcinomas of various other sites. ${ }^{13}$

Distant metastases of malignant salivary gland tumors may pose significant difficulties in their differential diagnosis from primary or other metastatic carcinomas. ${ }^{17}$ Knowledge of the CK7/CK20 immunophenotype of these tumors may prove valuable in their definitive diagnosis, especially in the absence of a previous diagnosis of a primary malignant salivary gland tumors. Although our study did not include metastatic malignant salivary gland tumors, it is reasonable to expect that these tumors would retain the CK immunohistochemical profile of the corresponding primary tumors. This assumption is supported by previous observations suggesting that the CK7/CK20 expression profile of most types of carcinomas is to a large extent retained in their metastases. ${ }^{3}$ Nevertheless, future studies should confirm this notion by comparing the CK7 and CK20 expression in primary malignant salivary gland tumors and their corresponding metastases.

In previous studies, squamous cell carcinomas of diverse origin have been shown to be predominantly negative for both CK7 and CK20, with the exception of cervical squamous cell carcinomas, which exhibit a predominant CK7 + /CK20- immunophenotype. ${ }^{6,7}$ Head and neck squamous cell carcinomas conform to this rule; in the study of Chu et $a l^{6} 67 \%$ of studied head and neck squamous cell carcinomas were negative for both CK7 and CK20, only 27 and 6\% being CK7 +/CK20- and CK7-/CK20 +, respectively. Moreover, Regauer et $a l^{33}$ reported that carcinomas of the Waldeyer's ring area are negative for both CK7 and CK20, with the exception of a subset of tumors that express CK7, produce CK7 + cystic 'fluid-filled' nodal metastases, and appear to arise from ducts of minor salivary glands with only limited involvement of the surface epithelium, consistent with a role for CK7 as a marker for glandular origin. Our results in squamous cell carcinomas secondarily involving salivary glands largely confirm these previous studies, in that most tumors are negative for both CK7 and CK20, the rest being weakly focally positive only for CK7. Given the relative frequency of squamous cell carcinomas involving major and minor salivary glands, as a result of metastasis or direct extension from adjacent sites, and the possibility of confusion between squamous cell carcinomas and malignant salivary gland tumors, especially high-grade mucoepidermoid carcinomas, ${ }^{19}$ the differential CK7/CK20 immunoprofile of these tumors can facilitate their discrimination. In this regard, the negative or focally and weakly positive CK7 staining in squamous cell carcinomas contrasts with the uniformly strong and 
predominantly diffuse CK7 immunoreactivity of mucoepidermoid carcinomas, which persists among high-grade tumors.

In conclusion, knowledge and assessment of the CK7/CK20 immunohistochemical profile of malignant salivary gland tumors, although of limited usefulness in the distinction between the various histologic subtypes of these tumors, may offer considerable aid in their differential diagnosis from numerous tumors of diverse origin, including squamous cell carcinomas.

\section{References}

1 Moll R, Franke WW, Schiller DL, et al. The catalog of human cytokeratins: patterns of expression in normal epithelia, tumors and cultured cells. Cell 1982; 31:11-24.

2 Moll R. Cytokeratins as markers of differentiation in the diagnosis of epithelial tumors. Subcell Biochem 1998;31:205-262.

3 Tot T. Cytokeratins 20 and 7 as biomarkers: usefulness in discriminating primary from metastatic adenocarcinoma. Eur J Cancer 2002;38:758-763.

4 Ramaekers F, van Niekerk C, Poels L, et al. Use of monoclonal antibodies to keratin 7 in the differential diagnosis of adenocarcinomas. Am J Pathol 1990;136: 641-655.

5 Wang NP, Zee S, Zarbo RJ, et al. Coordinate expression of cytokeratins 7 and 20 defines unique subsets of carcinomas. Appl Immunohistochem 1995;3: 99-107.

6 Chu P, Wu E, Weiss LM. Cytokeratin 7 and cytokeratin 20 expression in epithelial neoplasms: a survey of 435 cases. Mod Pathol 2000;13:962-972.

7 Moll R, Lowe A, Laufer J, et al. Cytokeratin 20 in human carcinomas. A new histodiagnostic marker detected by monoclonal antibodies. Am J Pathol 1992;140:427-447.

8 Miettinen M. Keratin 20: immunohistochemical marker for gastrointestinal, urothelial, and Merkel cell carcinomas. Mod Pathol 1995;8:384-388.

9 van de Molengraft FJ, van Niekerk CC, Jap PH, et al. OV-TL 12/30 (keratin 7 antibody) is a marker of glandular differentiation in lung cancer. Histopathology 1993;22:35-38.

10 Wauters CC, Smedts F, Gerrits LG, et al. Keratins 7 and 20 as diagnostic markers of carcinomas metastatic to the ovary. Hum Pathol 1995;26:852-855.

11 Loy TS, Calaluce RD. Utility of cytokeratin immunostaining in separating pulmonary adenocarcinomas from colonic adenocarcinomas. Am J Clin Pathol 1994;102:764-767.

12 Kaufmann O, Deidesheimer T, Muehlenberg M, et al. Immunohistochemical differentiation of metastatic breast carcinomas from metastatic adenocarcinomas of other common primary sites. Histopathology 1996;29:233-240.

13 Chan JK, Suster S, Wenig BM, et al. Cytokeratin 20 immunoreactivity distinguishes Merkel cell (primary cutaneous neuroendocrine) carcinomas and salivary gland small cell carcinomas from small cell carcinomas of various sites. Am J Surg Pathol 1997;21: 226-234.
14 Tot T. Adenocarcinomas metastatic to the liver: the value of cytokeratins 20 and 7 in the search for unknown primary tumors. Cancer 1999;85: 171-177.

15 Tot T. The value of cytokeratins 20 and 7 in discriminating metastatic adenocarcinomas from pleural mesotheliomas. Cancer 2001;92:2727-2732.

16 Duval JV, Savas L, Banner BF. Expression of cytokeratins 7 and 20 in carcinomas of the extrahepatic biliary tract, pancreas, and gallbladder. Arch Pathol Lab Med 2000;124:1196-1200.

17 Bradley PJ. Distant metastases from salivary glands cancer. ORL J Otorhinolaryngol Relat Spec 2001;63:233-242.

18 Seifert G, Hennings K, Caselitz J. Metastatic tumors to the parotid and submandibular glands-analysis and differential diagnosis of 108 cases. Pathol Res Pract 1986;181:684-692.

19 Sobral AP, Loducca SV, Kowalski LP, et al. Immunohistochemical distinction of high-grade mucoepidermoid carcinoma and epidermoid carcinoma of the parotid region. Oral Oncol 2002;38: 437-440.

20 Ellis GL, Auclair PL. Tumors of the salivary glands. In: Rosai J, Sobin LH (eds). Atlas of Tumor Pathology, 3rd Series Vol. 17. Armed Forces Institute of Pathology: Washington, DC, 1995.

$21 \mathrm{Su} \mathrm{L}$, Morgan PR, Harrison DL, et al. Expression of keratin mRNAs and proteins in normal salivary epithelia and pleomorphic adenomas. J Pathol 1993;171:173-181.

$22 \mathrm{Li} \mathrm{C}$, Okamoto Y, Ohmura $\mathrm{H}$, et al. Expression of cytokeratins in Warthin's tumour (adenolymphoma) of parotid glands: specific detection of individual cytokeratin types by monoclonal antibodies. Eur J Cancer B Oral Oncol 1996;32B:352-358.

23 de Araujo VC, de Sousa SO. Expression of different keratins in salivary gland tumours. Eur J Cancer B Oral Oncol 1996;32B:14-18.

24 Foschini MP, Marucci G, Eusebi V. Low-grade mucoepidermoid carcinoma of salivary glands: characteristic immunohistochemical profile and evidence of striated duct differentiation. Virchows Arch 2002;440: 536-542.

25 Barrett AW, Cort EM, Patel P, et al. An immunohistological study of cytokeratin 20 in human and mammalian oral epithelium. Arch Oral Biol 2000;45: 879-887.

26 de Araujo VC, de Sousa SO, Carvalho YR, et al. Application of immunohistochemistry to the diagnosis of salivary gland tumors. Appl Immunohistochem Mol Morphol 2000;8:195-202.

27 Machado de Sousa SO, Soares de Araujo N, Correa L, et al. Immunohistochemical aspects of basal cell adenoma and canalicular adenoma of salivary glands. Oral Oncol 2001;37:365-368.

28 Lewis JE, Olsen KD, Sebo TJ. Carcinoma ex pleomorphic adenoma: pathologic analysis of 73 cases. Hum Pathol 2001;32:596-604.

29 Krogdahl AS, Schou C. Mucinous adenocarcinoma of the sublingual gland. J Oral Pathol Med 1997;26: 198-200.

30 Araujo VC, Loducca SV, Sousa SO, et al. The cribriform features of adenoid cystic carcinoma and polymorphous low-grade adenocarcinoma: cytokeratin and integrin expression. Ann Diagn Pathol 2001;5: $330-334$. 
31 de Araujo VC, Kowalski LP, Soares F, et al. Salivary duct carcinoma: cytokeratin 14 as a marker of in situ intraductal growth. Histopathology 2002;41:244-249.

32 Darling MR, Schneider JW, Phillips VM. Polymorphous low-grade adenocarcinoma and adenoid cystic carcinoma: a review and comparison of immunohistochemical markers. Oral Oncol 2002;38:641-645.

33 Regauer S, Beham A, Mannweiler S. CK7 expression in carcinomas of the Waldeyer's ring area. Hum Pathol 2000;31:1096-1101. 